Revista de Urbanismo N³5 - Diciembre 2016 Departamento de Urbanismo - FAU - Universidad de Chile

\title{
EDITORIAL
}

\section{Cambios socios espaciales y tamaño de centros urbanos latinoamericanos}

No cabe duda que nos encontramos en un proceso urbano de mutación socio espacial que afecta al territorio global $y$, que en nuestro continente latinoamericano, se ve expresado de manera disímil, considerando la diversidad geográfica y cultural que ha sido cubierta por la academia (Almandoz, 2006). Sin embargo, hay elementos comunes que parecen persistir como parte del legado hispanoamericano: la cuadrícula que se extiende y pierde su forma original, la disputa del suelo urbano por sobre el rural, la densificación mal entendida como verticalización (Inzulza-Contardo, 2016) y los procesos de segregación que recambian barrios y pueblos aceleradamente (Borsdorf et al., 2016). Estos son los fenómenos que hemos querido retratar en el presente número de Revista de Urbanismo, a través de lo que hemos denominado, "cambios socios espaciales y tamaño de centros urbanos latinoamericanos". Para ello, presentamos diez trabajos de calidad, en que se discuten problemáticas derivadas de la densificación urbana, la dispersión y los constantes cambios en la morfología de las ciudades (Janoschka, 2002). Abrimos los fuegos con el tema de la compacidad urbana y su estrategia metodológica, tratado en el escrito deMarielisa Quintero Bosetti. Por medio de un modelo de análisis operacional, la autora aporta con "criterios de diseño dirigidos a la consolidación de ciudades compactas, sostenibles en lo social, en lo económico y en lo ambiental, y que propendan a la cohesión urbana". Este modelo es instructivo e introductorio para entender las complejidades de la ordenación urbana y ambiental que se aplican en los centros urbanos incluidos en este número.

Posteriormente, se presentan tres grupos de investigaciones como una forma de "trabajo en terreno" por nuestro continente y ordenados en temáticas especificas. El primer grupo inicia un debate refrescado de cuatro trabajos que examinan fenómenos contemporáneos de cambio socio espacial, y en particular, acentúan su análisis en la producción de vivienda en su amplia dimensión. Alfredo Corona Consuelo, Carlos Garrocho Rangel y Juan Campos Alanís presentan un análisis de segregación residencial de adultos mayores en la Zona Metropolitana del Valle de Toluca, México, un estudio contundente sobre la movilidad de la población adulta mayor que reside en el centro de la ciudad, a pesar del cambio de funcionalidad de éste. Esta localización y búsqueda por la ciudad central (inner city) se puede extrapolar en la otra "cara de la moneda" con el trabajo de Lina Machado Magalhaes, quien investiga acerca de la producción elitista del espacio urbano en Rio de Janeiro, Brasil. Mediante el análisis del proceso de producción social en dicha ciudad, la autora sostiene que la segregación socio-espacial es "resultado de un proceso histórico del desarrollo de un urbanismo clasista, materializado en las reformas urbanas del inicio del siglo XX". De esta forma, la informalidad característica $\mathrm{Y}$ controversial de nuestras urbes latinoamericanas representadas por las favelas, villas miserias, barriadas o poblaciones callampas, versus la centralidad que proyectos del tipo waterfont como Porto Maravilha, parecen mantenerse como los grandes dilemas a enfrentar por la planificación urbana contemporánea.

Luego, Juan Jiménez Caldera presenta una metodología para la medición del déficit cualitativo de espacio público, aportando con ello una herramienta para el ordenamiento del territorio, que contribuye llenando los vacíos y debilidades de la normativa existente en el contexto colombiano, y que es además claramente replicable en el resto de nuestro continente. Mediante una matriz multi atributos - elementos del espacio público, escala vecinal, zonal o regional, componentes ambientales, mobiliario y planes para la potenciación, creación y recuperación - el autor devela la carencia de equipamiento urbano en los espacios próximos 
del hábitat residencial. Este análisis sobre el panorama deleznable de atributos del entorno residencial, es posible extrapolarlo además al centro de Guayaquill, con el análisis sobre el desarrollo de viviendas de interés social y la recuperación de plusvalías en ésta área, presentada por Alina Delgado Bohorquez. La autora entrega un análisis sobre las estrategias de gestión territorial que se pueden aplicar para el financiamiento de proyectos de vivienda social en el centro de la ciudad de Guayaquil, utilizando propuestas urbanas conceptuales y enmarcándolas dentro de la discusión del derecho a la ciudad. Resulta interesante destacar que la "utilización de estos instrumentos se podría al mismo tiempo que atraer inversiones al centro, asegurar la inclusión de vivienda social, mejorar la calidad de los espacios públicos, y contribuir a recuperar la memoria histórica del centro".

Sin duda, este intento de integración, que es probablemente el mayor desafío del urbanismo táctico y social, con visiones encontradas "de las elites y de los grupos de menores ingresos", va de la mano con la morfología y escala de la ciudad con sus sistemas de asentamientos humanos. Desde esta óptica más bien formal, se entrelazan dos trabajos del segundo grupo, resaltando la escala de ciudad de menor tamaño, por medio de la vocación rural que se articula en un sistema urbano y viceversa. En primer lugar, a través del concepto de "rura-ciudades" como alternativa de planeación urbana inteligente, abogado por los autores Silverio Hernández Moreno, José Hernández Moreno y Bianca Alcaraz Vargas, se propone "un cambio de paradigma" relacionado con la planeación de ciudades, en donde "se resalta el empleo de los usos de suelo de tipo rural complementados con usos de suelo de tipo urbano, los cuales se funden en un híbrido que mejora el metabolismo urbano de las ciudades". Mediante este argumento demostrado en el caso mexicano - incluyendo Ciudad de México, Valle de México, Puebla, Toluca, Pachuca, Cuernavaca, Cuautla y Tlaxcala - los autores tensionan el rol de las políticas de gestión y planeación del uso de suelo, que parecen alejarse de una imagen objetivo clara en el crecimiento urbano y, más aún, de la inclusión de etapas de evaluación estratégica de mediano y largo plazo.

De esta forma, el deterioro y fragmentación del medio rural y la disminución de los distintos impactos ambientales causados por la urbanización coetánea, también son confrontados en el análisis del contexto chileno por las autoras Marcela Soto Caro y Anne Escobar Guegen, por medio del estudio sobre el valor del suelo agrícola en la ciudad de Chillán, considerada como de escala intermedia. Se señala enfáticamente que "el desarrollo de nuevos factores y condiciones que van consolidando los procesos de crecimiento y transformación urbana desigual, bajo el modelo neoliberal imperante y persistente, se hacen cada vez más visibles en las ciudades de rango intermedio". Es por ello que la clásica relación emparentada "forma y función" sigue con mayor vigencia si consideramos la "dicotomía que enfrentan las ciudades de rango medio que tienen como base productiva la agricultura y por otro lado la dependencia a mercados financieros y comerciales que promueven el consumo de suelo urbano". Dentro de esta relación, finalmente, se agrupan tres trabajos que adicionan además la importancia de las condiciones del riesgo tanto natural como urbano, implícitos en la planificación urbana y, que por tanto, requieren de un análisis tanto de sus elementos basales de emplazamiento, como de su atributos culturales de habitar la pendiente, el volcán y el plano.

Ejemplo de lo anterior, es la puesta en valor de la reestructuración urbana de Valparaíso, Chile, en base a sus valores patrimoniales latentes, por medio del trabajo de Eliana Muga Weippert y Paz Carreño Zunino. Mediante una metodología de investigación teórico/práctica, el estudio se adentra en la forma de habitar la pendiente, buscando con ello "revertir los procesos de marginalidad, asociados a diversos problemas ambientales como micro-basurales, ocupación ilegal de terrenos y peligro de incendios, a través de la generación de nuevas centralidades urbanas y el cuidado de las quebradas con su ocupación y manejo". Este esfuerzo de entender la "territorialidad" del espacio urbano y sus riesgos asociados es compartido en el trabajo de 
Erasmo Aguilar Arriola, sobre la importancia de la vulnerabilidad estructural de la vivienda unifamiliar en el riesgo urbano. Para ello, el autor nos retrata como la vivienda unifamiliar, que es el "nodo vital" de Managua en Nicaragua, especialmente los de viviendas populares o de interés social, se desarrolla en muchas ocasiones junto a "las amenazas naturales y las particularidades propias de la ciudad más poblada y con mayor afluencia de usuarios", creando un alto nivel de riesgo urbano. Es por ello que se aboga por la necesidad de una metodología de estudio apropiada, que incluya indicadores sobre la "salud" de la estructura de la vivienda, como se presentan en esta investigación.

Por su parte, el riesgo visto desde otra esfera, se pone en conocimiento mediante el desplazamiento de familias de bajos ingresos del centro de Talca hacia la periferia. Los autores

Francisco Letelier Troncoso y Alejandra Rasse Figueroa retratan como el terremoto de $8.8^{\circ}$ en la escala de Richter, que afectó la zona centro sur de Chile el 27 de febrero de 2010, terminó por perjudicar y hacer vulnerable la permanencia que aquellos que no eran propietarios, así como su condición socioeconómica y la prevalencia de la jefatura de hogar femenina y de adultos mayores. Agregan que "este riesgo no fue abordado por las políticas públicas, que carecieron de perspectiva territorial y consideración del derecho a la localización".

De esta forma, los diez trabajos que se presentan, y con buena producción de citación de revistas Scopus e ISI a la par con los nuevos trazos de Revista de Urbanismo, permiten epilogar sobre la necesidad de amplificar la visión de la planificación urbana frente a las singularidades de nuestro continente latinoamericano, y con mayor cuidado en recapturar las ciudades para su gente (Roberts, 2005; De Souza, 2009). Solo a través de esta vía de integración se podrá hacer frente a la segregación social, ampliamente discutida desde la academia y la práctica profesional (Rodríguez,2008).

\section{Referencias bibliográficas}

Almandoz, A. (2006) Urban planning and historiography in Latin America, Progress in Planning, 65(2), $81-123$.

Borsdorf, A., Hidaldo, R., Vidal-Hoppmann, S. (2016). Social segregation and gated communities in Santiago de Chile and Buenos Aires. A comparison. Habitat International 54, 18-27.

De Souza, M. L. (2009). Cities for people, not for profit-from a radical-libertarian and Latin American perspective. City 13(4), 483-492

Janoschka, M. (2002). El nuevo modelo de la ciudad latinoamericana: fragmentación y privatización. Eure, $85,11-20$.

Rodríguez, J. (2008). Dinámica sociodemográflca metropolitana y segregación residencial: ¿qué aporta la CASEN 2006? Revista de Geografía Norte Grande, 41, 81-102

Inzulza-Contardo, J. (2016). Contemporary Latin American gentrification? Young urban professionals (YUPs) discovering historic neighbourhoods, Urban Geography 37(8), 1195-1214

Roberts, B R. (2005). Globalization and Latin American cities. International Journal of Urban and Regional Research 29(1), 110-123. 